\title{
MUJER, NIÑOS Y VARIABLES PSICOSOCIALES EN EL FÚTBOL ESPAÑOL. UNA REVISIÓN BIBLIOGRÁFICA DE LOS AÑOS 2015-2019
}

\author{
MULHERES, CRIANÇAS E VARIÁVEIS PSICOSSOCIAIS NO FUTEBOL \\ ESPANHOL. UMA REVISÃO BIBLIOGRÁFICA DOS ANOS 2015-2019
}

\author{
WOMEN, CHILDREN AND PSYCHOSOCIAL VARIABLES IN SPANISH \\ FOOTBALL. A BIBLIOGRAPHIC REVIEW OF THE 2015-2019 PERIOD
}

\author{
Alberto Sanmiguel-Rodríguez*, Víctor Arufe Giráldez*
}

Palabras clave: Mujeres.

Niños.

Fútbol.

Revisión.

Resumen: El fútbol es un fenómeno socio cultural que acapara la atención de una gran parte de la población. El objetivo de este trabajo es realizar una revisión de la literatura científica de los últimos cinco años que aborde el ámbito del fútbol español, seleccionando para el análisis de contenido solamente los artículos relacionados con la participación de la mujer, de los niños y aquellos que aborden variables psicosociales. Se ha realizado una exhaustiva revisión en la que se encontraron 212 documentos, reduciéndose a 54 manuscritos que cumplían con los criterios de inclusión. Como conclusiones se detecta un número escaso de publicaciones que abordan mujer y fútbol. Los artículos sobre fútbol y niños destacan los beneficios de esta práctica para el desarrollo fisiológico, físico y para la salud. Finalmente, se concluye que las variables psicosociales más estudiadas en el fútbol han sido la motivación, la autoeficacia, ansiedad, cohesión grupal y autoconcepto.

Palavras chave Mulheres. Crianças. Futebol. Revisão.
Keywords: Women. Child. Football. Review.
Resumo: $O$ futebol é um fenômeno sociocultural que chama a atenção de grande parte da população. $O$ objetivo deste trabalho é fazer uma revisão da literatura científica dos últimos cinco anos que aborda o campo do futebol espanhol, selecionando para análise de conteúdo apenas artigos relacionados à participação de mulheres, crianças e aqueles que abordam variáveis psicossociais. Foi realizada uma revisão exaustiva na qual foram encontrados 212 documentos, reduzidos a 54 manuscritos que atendiam aos critérios previamente estabelecidos. Como conclusão, um pequeno número de publicações sobre mulheres e futebol é detectado. Os artigos sobre futebol e crianças incluem os benefícios dessa prática para o desenvolvimento fisiológico, físico e de saúde. Por fim, conclui-se que as variáveis psicossociais mais estudadas no futebol foram motivação, autoeficácia, ansiedade, coesão grupal e autoconceito

\footnotetext{
Abstract: Football is a socio-cultural phenomenon that captures the attention of a large part of the population. This work reviews scientific literature of the last 5 years addressing the field of Spanish football, covering only articles related to the participation of women, children and those that address psychosocial variables. An exhaustive review has been carried out in which 212 documents were found. After exhaustively reading, they were reduced to 54 manuscripts that met previously established criteria. As a conclusion, a small number of publications on women and football were detected. Articles about football and children include the benefits of this practice for physiological, physical and health development. Finally, the most studied psychosocial variables in football were motivation, self-efficacy, anxiety, group cohesion and self-concept.
}

*Universidad de Vigo. Pontevedra. España.

E-mail: asrgz2014@gmail.com

**Universidad de A Coruña. A Coruña. España.

E-mail: varufe@yahoo.es

Recebido em: 22-04-2019 Aprovado em: 22-10-2019 Publicado em: 20-12-2019

DOI: https://doi.org/10.22456/1982-8918.92077 (c) (1) (8) Licence 


\section{INTRODUCCIÓN}

El fútbol es uno de los deportes que más atención acapara por parte de la población a nivel mundial. Algunos autores señalan que más que un deporte es un negocio que ofrece un rendimiento excepcional para los participantes en este modelo de negocio (PRADO-ROMÁN et al., 2017). Sin embargo, en los últimos años parece que la situación económica del deporte español no es tan prominente como antes y que han comenzado a detectar disminuciones del público en los estadios y reducción del número de aficionados (PRADO-ROMÁN et al., 2017). El fútbol es un juego de estrategia concurrente multijugador, uno de los deportes más populares del mundo. Cada partido de fútbol es un fenómeno social por sí mismo, con un impacto social de alto nivel desde instancias locales hasta internacionales (TÉLLEZ-GIRÓN; ALVARADO, 2016). El fútbol es un deporte y trabajo en equipo "de ritmo externo", que requiere "habilidades de función ejecutiva" (LANDERS; ESCH, 2015).

La evolución del fútbol en los últimos 30 años ha generado un ritmo de juego más rápido y dinámicas de jugadores más exigentes que han llevado a un cambio de paradigma en la forma en que se analiza el juego. Los estudios clásicos, puramente descriptivos, pintan una imagen inconexa que está lejos de la realidad de la compleja red de relaciones e interacciones que marcan el fútbol moderno (MANEIRO; AMATRIA; ANGUERA, 2019). El fútbol de hoy se ha convertido en un reemplazo de la religión, no solo como un hecho trascendente sino también como un hecho real. El alcance mundial que ha logrado el fútbol en los últimos años nos lleva a creer que el hombre contemporáneo ha hecho de los equipos de fútbol su objeto de adoración, creencias y anhelo de felicidad (ALBÓ, 2017). Para López-Martínez y Fernández-Fernández (2015) la prominencia que el fútbol ha ganado en las últimas décadas, ha transformado este tipo de actividad en un fenómeno de gran impacto socioeconómico que va más allá de lo que es simplemente el deporte. Sin embargo, uno de sus aspectos clave, los clubes, se han convertido en verdaderas empresas que, en la mayoría de los casos, no se gestionan bajo los imperativos legales y éticos que conlleva el resto de entidades comerciales.

En las últimas décadas, el fútbol ha sido analizado con gran detalle, lo que permite mejorar los aspectos técnicos y tácticos del juego a través de la observación, el análisis y el entrenamiento (DÍAZ-CIDONCHA et al., 2015). Hoy en día las estadísticas relacionadas con los juegos en la industria del fútbol son exigidas por entrenadores, jugadores, gerentes, periodistas, seguidores, fanáticos, desarrolladores de videojuegos, mercados de apuestas y académicos. Sin embargo, el empleo de estadísticas relacionadas con el juego para analizar el rendimiento en el fútbol tiene problemas inherentes dado que es un fenómeno multifacético y complejo (ZAMBOM-FERRARESI; RIOS; LERA-LÓPEZ, 2018).

Según Sarmento et al., (2018) muchos investigadores están estudiando repetidamente las especificidades de cómo se logra la excelencia en el fútbol, un deporte practicado en todo el mundo, a través de una variedad de enfoques y disciplinas científicas. Los resultados de su trabajo indican que los jugadores más exitosos presentan ventajas técnicas, tácticas, antropométricas, fisiológicas y psicológicas que cambian con el estado de maduración y las posiciones de juego. Estos hallazgos deben ser considerados cuidadosamente por aquellos involucrados en la identificación y búsqueda de jugadores de fútbol. Actualmente faltan investigaciones que aborden los aspectos psicológicos y ambientales que influyen en la identificación y el desarrollo del talento en el fútbol (SARMENTO et al., 2018). Para Maneiro y 
Amatria (2018) la investigación en el fútbol se ha centrado tradicionalmente en aspectos muy específicos del juego, como los aspectos técnicos y fisiológicos, y ha ignorado en gran medida cuestiones importantes como el rendimiento táctico y el papel de los jugadores individuales dentro del equipo. Hevilla-Merino y Castillo-Rodríguez (2018) señalan que la competencia en el fútbol supone que el deportista se enfrenta a situaciones de máxima activación que, en varias ocasiones, pueden causar cambios en sus características psicológicas y, en consecuencia, influir en su rendimiento deportivo. Atendiendo al trabajo de Aguilar (2018) la optimización del rendimiento deportivo de alto nivel requiere nuevas perspectivas analíticas para comprender cómo surge el éxito en el fútbol. Caballero, Rubio y Godoy (2017) muestran la importancia que tienen la ubicación del partido, el marcar primero, la calidad del equipo rival, las sustituciones y las cartas en el resultado del partido, mientras que la regresión lineal resalta el efecto anotar primero, la calidad del equipo rival y las sustituciones en la clasificación final del equipo.

Vaczi (2015) expone que el fútbol español está en la cima del mundo, a nivel internacional y de clubes, con los mejores equipos y un suministro aparentemente infinito de jugadores emocionantes y con clase mundial. Mientras la economía española tambalea, su fútbol florece, profundamente arraigado a lo largo de la vida social y cultural española. Otros autores (MOSCOSO-SÁNCHEZ; RODRÍGUEZ-DÍAZ; FERNÁNDEZ-GAVIRA, 2015) analizaron la situación ambivalente del deporte en España, como resultado del predominio de una doble moral de deporte de élite y deporte para todos. La administración española prioriza las políticas para el deporte de élite y éstas dificultan el desarrollo de deportes de base y deportes para todos. Los resultados de su investigación (MOSCOSO-SÁNCHEZ et al., 2015) muestran que, a pesar de los éxitos y el triunfalismo de los medios nacionales con respecto a los deportes competitivos en España, gracias a las victorias de atletas famosos, clubes deportivos y la selección nacional de fútbol, la realidad de la práctica del deporte en España es muy diferente. La mayoría de la población no practica deporte, la mayoría de las instalaciones deportivas se construyeron antes de 1995 y, por último, la política deportiva, centrada en el deporte y el entretenimiento de alto rendimiento, es la causa de una brecha deportiva significativa (MOSCOSO-SÁNCHEZ et al., 2015). En otro orden de ideas, SIMÓN (2018) investigó el papel desempeñado por el fútbol como un medio para construir, configurar y fortalecer las identidades nacionales en España entre 1975 y 2017. Para Bunk (2015) los españoles en la ciudad de Nueva York durante la década de 1920 utilizaron el fútbol para crear y mantener múltiples nociones de identidades centradas en afiliaciones supranacionales, nacionales y regionales. El éxito en el campo contra otros equipos demostró el talento y la fuerza no solo de los españoles sino también de los inmigrantes del resto del mundo hispanohablante. El deporte también generó redes que reforzaron una identidad nacional española al conectar a los neoyorquinos con comunidades similares tanto en los Estados Unidos como en el extranjero (BUNK, 2015).

El fútbol femenino español poco a poco va dando sus pasos para alcanzar un mayor protagonismo social y una mayor profesionalización. Fruto de este escaso reconocimiento tenemos la hipótesis de que la producción científica también será baja. Autores que han abordado la investigación en el fútbol femenino en otros países confirman también la escasez de reconocimiento científico y profesional al fútbol femenino (GONZÁLEZ; FERNÁNDEZORTEGA; GARAVITO-PEÑA, 2019; SOUZA; ESPIRITO SANTO; RIBEIRO, 2018). Todos los agentes implicados en el sistema deportivo deben luchar por una visibilidad de género responsable e igualitaria. Las conclusiones de las Jornadas Nacionales sobre el acceso de las mujeres al deporte profesional: el caso del fútbol celebradas en Madrid el 19 y 20 de 
abril del 2018 (GÓMEZ; ALFARO, 2018) son claras y tajantes confirmando la persistencia de una subordinación cultural y social de la mujer al hombre que tiene también su reflejo en el ámbito deportivo. Señalan además que la educación y los cambios socioculturales se revelan como necesidades para lograr la igualdad de oportunidades entre mujeres y hombres. Y destacan el bajo favorecimiento de la incorporación de mujeres, tanto deportistas como directivas y técnicas, a las estructuras administrativas y organizativas del deporte, confirmando la presencia de estereotipos de género en el deporte y especialmente en el fútbol donde se considera que ha habido pocos cambios y se mantiene un claro predominio de la práctica y consideración masculina. Uno de los motivos que confirma la baja práctica femenina se encuentra en que solamente el $5 \%$ del total de fichas federativas de futbol español son de mujeres. Las conclusiones finalizan señalando que a pesar del desequilibrio existente en la práctica, el fútbol femenino ha tenido un avance en los últimos años que se manifiesta tanto a nivel internacional como nacional. Sin embargo, todavía las jugadoras, entrenadoras y árbitras que quieren progresar tienen que emigrar al extranjero.

Por todo lo anteriormente comentado, el objetivo de este trabajo es realizar una revisión de la literatura científica de los últimos 5 años (2015-2019) que aborde el ámbito del fútbol español seleccionando para el análisis de contenido solamente los artículos relacionados con la participación de la mujer, la participación de los niños y por último los que aborden variables psicosociales.

\section{METODOLOGÍA}

La revisión bibliográfica es un tipo de artículo científico que sin ser original recopila la información más relevante sobre un tema específico (GUIRAO-GORIS; OLMEDO; FERRER, 2008). Para esta revisión se ha realizado una búsqueda bibliográfica en dos importantes bases de datos, una inglesa Scopus y otra española Dialnet. Además se utilizó también el buscador internacional Google Scholar que rastrea e incorpora a su depósito todos los artículos publicados en internet. Como palabras clave se eligieron del Tesaurus de la UNESCO los siguientes términos [soccer, football, fútbol, Spanish, español]. Los criterios de inclusión utilizados fueron los siguientes:

- Artículos científicos publicados entre el año 2015 y el día 31 de marzo del 2019.

- Que aborden cualquier tipo de investigación vinculada al fútbol en el contexto español, incorporando estudios experimentales, descriptivos, cuasi-experimentales y/o estudios de caso.

- Que estén publicados en español o inglés.

Tras aplicar estos criterios la selección final congregó a un total de 212 documentos que han sido publicados en inglés y español desde el año 2015 hasta marzo del año 2019.

El cronograma de trabajo para la búsqueda de la información tuvo cuatro fases diferenciadas: 1ํ Fase: Búsqueda y selección de los descriptores a través del Thesaurus de la UNESCO. 2ª Fase: Búsqueda pormenorizada en las bases de datos científicas SCOPUS y Dialnet y en el buscador Google Scholar utilizando los criterios de inclusión descritos anteriormente. $3^{3}$ Fase. Análisis del contenido de los artículos y separación por temáticas. 4aㅡ Fase. Categorización de los artículos y elaboración del manuscrito.

Tras la revisión de los 212 documentos se descartaron aquellos artículos que no abordasen directamente la presencia de la mujer en el fútbol español, niños y fútbol español 
y/o estudios vinculados al análisis de las variables psicosociales en el fútbol español. Después de este filtro se computaron un total de 54 manuscritos que aportasen calidad metodológica y cumpliesen con los criterios de inclusión relatados anteriormente, quedando el reparto de artículos entre las tres categorías establecido de la siguiente forma:

Tabla 1 - Categorías de análisis de la literatura científica y número de artículos publicados

\begin{tabular}{lc}
\hline Categoría & Número de artículos \\
\hline Investigaciones relacionadas con el fútbol femenino español & 8 \\
\hline $\begin{array}{l}\text { Investigaciones relacionadas con el fútbol de base, amateur, detección de talento } \\
\text { y deporte escolar en España }\end{array}$ & 27 \\
\hline $\begin{array}{l}\text { Investigaciones relacionadas con motivación, cohesión grupal, estrés y ansiedad } \\
\text { en el fútbol español }\end{array}$ & 19 \\
\hline
\end{tabular}

Fuente: elaboración propia

\section{RESULTADOS Y DISCUSIÓN}

Se ha optado por agrupar los resultados de esta revisión en las 3 grandes categorías contempladas anteriormente y comentar las principales conclusiones y resultados de cada uno de los manuscritos revisados.

\subsection{INVESTIGACIONES RELACIONADAS CON EL FÚTBOL FEMENINO}

Llopis-Goig y Flores (2017) abordan en su artículo el estudio de las peñas femeninas en el fútbol español destacando el gran interés socio-antropológico, ya que permite una mejor comprensión de la dinámica que opera en la dimensión de género de este deporte. Señalar que las peñas son grupos de personas unidas con un mismo fin, en el contexto de este artículo su significado es el de cultivar una afición y fomentar y apoyar a un equipo de fútbol femenino. A través de una entrevista realizada a una muestra de siete mujeres pertenecientes exclusivamente a peñas de fútbol femenino señalan que las entrevistadas perciben su pertenencia a la peña como un espacio de liberación de la dominación masculina del fútbol. Por otro lado, estas mujeres sienten que estas peñas les permiten afirmar su interés por este deporte y crear su propio espacio a su alrededor. Los autores señalan que las peñas femeninas se convierten en una vía de acceso al espacio público del fútbol fuertemente masculinizado y en un medio para alcanzar una posición de reconocimiento en este espacio

Zambom-Ferraresi, García-Cebrián y Lera-López (2018) analizaron el equilibrio competitivo en las dos ligas españolas de fútbol, la liga masculina y la liga femenina estudiando el rendimiento de todos los equipos que la integran a lo largo de cuatro temporadas (de 2012/13 a 2017/18). Los resultados indicaron que la Liga Santander (masculina) es más equilibrada que la Liga Iberdrola (femenina). Sin embargo, estos resultados no implican que Liga Santander esté equilibrada. Los autores concluyen que a pesar del reciente aumento de medios de comunicación y asistencia en la Liga Iberdrola, la falta del equilibrio competitivo podría ser un serio obstáculo para que se convierta en una liga profesional.

Pérez et al. (2018) trataron de comprender las razones que llevan a las mujeres a jugar al fútbol. Los resultados de esta investigación indican que por norma general, a las futbolistas 
les gusta este deporte. Asimismo, señalan que para aumentar la motivación, es necesario llevar a cabo entrenamientos que tengan un alto contenido de diversión y trabajo en equipo, y que supongan un reto para los jugadores más veteranos. Sedano, Vaeyens y Redondo (2015) en un estudio realizado en una muestra de 4035 jugadoras analizaron la edad relativa, es decir cómo la fecha de nacimiento de una jugadora puede contribuir a la excelencia deportiva ocasionando diversas consecuencias que esta diferencia tiene sobre los sujetos y conocidas como el Efecto Relativo de la Edad (RAE). El trabajo analiza los efectos relativos de la edad en el fútbol femenino español e identifica la influencia de una posición de juego. Los autores concluyen que en la estructura actual del fútbol femenino español existe un efecto relativo de la edad, probablemente debido a los primeros procesos de identificación del talento.

Por otro lado, Pascual y Carbonell (2018) analizaron los aspectos técnicos y tácticos durante 30 partidos de fútbol femenino de primera división durante la temporada 2016-2017 de la liga Iberdrola. Las acciones técnicas realizadas con mayor frecuencia fueron pases y control de balón; en cambio los menos frecuentes fueron las acciones defensivas, habilidades y fintas. Por su parte, Ibáñez et al. (2018) estudiaron el impacto de anotar primero en el resultado final del partido y el desempeño de los equipos de fútbol profesional femenino. Cuanto mejor sea la posición en la clasificación, mayores serán las posibilidades de anotar primero. No observaron influencia de la ubicación del juego, la calidad de la oposición y la superficie del terreno de juego. Al marcar primero, los equipos clasificados del $1^{\circ}$ al $3^{\circ}$ obtienen la victoria en más del $90 \%$ de las veces, mientras que los equipos del $3^{\circ}$ al $10^{\circ}$ en casi el $80 \%$. A su vez, los equipos clasificados en el puesto 11 al 14 redujeron a $54 \%$ las posibilidades de ganar, incluso si anotaban primero.

Por otra parte, hay investigadores que estudiaron las lesiones en el fútbol femenino. Para Del Coso, Herrero y Salinero (2018) conocer la incidencia, el tipo, la ubicación y la gravedad de las lesiones causadas en el fútbol femenino español y los factores de riesgo para sufrir una lesión deportiva es el primer paso para desarrollar acciones preventivas. La mayoría de las lesiones fueron en las extremidades inferiores $(74,0 \%)$, afectando principalmente la rodilla $(30,4 \%)$ y las articulaciones del tobillo $(17,9 \%)$. También encontraron una mayor incidencia de lesiones en jugadoras de fútbol adultas de 18 años en adelante, en comparación con las menores de 18 años. Gutiérrez et al. (2018) evaluaron la extensibilidad de los isquiotibiales en jugadoras de fútbol femenino de élite. Los resultados de este estudio indican que la extensibilidad de los músculos isquiotibiales estudiados es limitada, por lo que se deberían implementar programas específicos de estiramiento de los músculos isquiotibiales en las mujeres en todas las categorías.

\subsection{INVESTIGACIONES RELACIONADAS CON EL FÚTBOL DE BASE, AMATEUR, DETECCIÓN DE TALENTO Y DEPORTE ESCOLAR}

La actividad física durante la infancia puede ser beneficiosa a largo plazo. Sin embargo, esta práctica puede influir en el desarrollo fisiológico del niño (DÍAZ-MIGUEL et al. 2018). Siguiendo estas líneas, Lozano-Berges et al. (2018) muestran que la práctica de fútbol durante el crecimiento puede mejorar la geometría ósea en jugadores de fútbol. Hellín, García-Jiménez y García-Pellicer (2019) indicaron que debido al aumento de la obesidad infantil, la Educación Física y los deportes como el fútbol, bádminton y los juegos motores se convierten en la mejor 
herramienta para combatir el sobrepeso entre los escolares. El porcentaje y tiempo dedicado de actividad física moderada a vigorosa son significativamente más altos para los juegos motores y fútbol en comparación con los juegos aeróbicos y bádminton. Las niñas obtuvieron un mayor porcentaje y tiempo de actividad física moderada a vigorosa que los niños. Asimismo, los valores más altos de frecuencia cardíaca se observaron en las lecciones sobre fútbol y habilidades motoras como actividad principal (HELLín et al., 2019). Lapresa et al. (2017) analizaron el impacto en el rendimiento técnico de los jugadores de 5 a 7 años de una unidad de enseñanza diseñada para garantizar el respeto en base a un conjunto de premisas que promueven la superioridad numérica del equipo en ataque para mejorar el rendimiento técnico de los jugadores. Por su lado, Castellano, Echeazarra y Estefano (2017) estudiaron qué dimensiones son adecuadas para cada edad, a pesar de la limitación del acondicionamiento físico. Para Serra-Olivares, García-López y Calderón (2017), el nivel de experiencia se correlacionó significativamente con el rendimiento del juego en las decisiones y ejecuciones tomadas por los jugadores más jóvenes. Los resultados de esta investigación (SERRA-OLIVARES et al., 2017) mostraron que la toma de decisiones aumentaron con el nivel de experiencia. Según Peña-González et al. (2018) la identificación del talento y el proceso de selección en jóvenes jugadores de fútbol masculino se centra principalmente en la antropometría y el rendimiento físico, pero los factores sociales también se consideran relevantes en este proceso y no se vieron afectadas por el año de nacimiento. Para García y Santana (2018) indican que, desde una edad temprana, se pueden encontrar algunas diferencias con respecto a la resistencia mental, según el nivel de rendimiento. Por su parte, Práxedes et al. (2017) muestran que los jugadores nacidos durante los primeros meses del año tienden a ser seleccionados para jugar en equipos con un nivel más alto de experiencia deportiva en cada categoría debido a su madurez física. En consecuencia, esto causa diferencias en cuanto a la experiencia que acumulan y la motivación que esto crea en estos jugadores.

Por otro lado, Balaguer et al. (2018) señalan la importancia del apoyo ofrecido por el entrenador y/o educador para promover la calidad de la experiencia de ocio de los jóvenes jugando al fútbol y sus beneficios para su bienestar. Así pues, Sevil et al. (2017) proponen un cambio en la configuración regulatoria de las categorías de jóvenes y niños que permita una adaptación progresiva a las habilidades y las necesidades de entrenamiento de los más jóvenes. Cejudo et al. (2019) y López-Valenciano et al. (2019) analizaron el trabajo de la flexibilidad en jóvenes futbolistas y refuerzan la necesidad de prescribir ejercicios dirigidos a mejorar la flexibilidad de cadera, rodilla y tobillo dentro de las rutinas diarias de entrenamiento de fútbol. En otro orden de ideas, Conejos et al. (2015) analizaron intervenciones efectivas, como el póster virtual o carteles e indican que éstos medios podrían ayudar a los jugadores de fútbol en el conocimiento nutricional que permitiría reducir la fatiga y a aumentar el rendimiento.

A su vez, Amatria et al. (2016) nos señalan que los formatos y partidos pequeños brindan a los niños mejores oportunidades para desarrollar sus habilidades y progresar como jugadores individuales y de equipo. Se encontró que la probabilidad de tiro al gol fue mayor en fútbol 7 que en fútbol 8, también tuvieron más oportunidades de controlar el balón, tirar a portería y tener más posibilidades de éxito en este formato. Asimismo, Clemente, Moreno-Arrones y Gil (2019) mostraron que, independientemente de la orientación del espacio de juego utilizado, el rendimiento de los jugadores disminuyó entre las series, demostrando la importancia de programar tiempos de recuperación adecuados entre las series para mantener las demandas físicas. Los resultados de la investigación llevada a cabo por Ortega-Toro et al. (2018) muestran que en los partidos de 
fútbol 5 , los porteros realizaron más acciones defensivas y acciones ofensivas que en los partidos de fútbol 8. La reducción en el tamaño del campo, la cantidad de jugadores y el tamaño de las porterías resultó que los porteros menores de 12 años tengan más interacción y más variabilidad en sus acciones. Siguiendo estas ideas, Casamichana, Bradley y Castellano (2018) demuestran que aumentar la longitud en lugar del ancho de 5 por lado tiene un mayor impacto en las respuestas de los jugadores en términos de aumentar las cargas de trabajo. Para Nevado-Garrosa et al. (2015) si bien la carga de trabajo total es mayor en la situación de $11 \times 11$, el $7 \times 7$ resulta más exigente en términos de aceleraciones y cambios de dirección. Además, las demandas físicas del $7 \times 7$ convierten a este formato en una tarea ideal para trabajar la fuerza específica, en un contexto donde se integran aspectos físicos, técnicos y tácticos. Otro estudio (LOS ARCOS et al., 2015) compararon los efectos de Small-Sided Games versus Interval Training en el entrenamiento de fútbol sobre el ejercicio aeróbico y el disfrute físico en jugadores de fútbol de élite juvenil. Los entrenadores podrían usar el entrenamiento de Small-Sided Games durante las últimas semanas de la temporada como una opción sin temor a perder la aptitud aeróbica y al mismo tiempo promover un alto disfrute físico de sus jugadores.

López-Fernández et al. (2018) muestran que el comportamiento mecánico de las superficies de césped natural y artificial no difiere lo suficiente como para causar diferentes respuestas fisiológicas y neuromusculares. Asimismo, Díaz-Cidoncha et al. (2015) revelaron que no hubo diferencias significativas para las diferentes superficies o formatos de juego. Azcarate, Los Arcos y Yanci (2018) analizaron durante 12 semanas la capacidad de aceleración, el cambio de dirección y la capacidad cardiovascular de jugadores de fútbol que compiten en la tercera división y regional preferente. Así pues, tanto la totalidad de los jugadores como el grupo de tercera división y preferente obtuvieron una mayor capacidad y velocidad aeróbica máxima. Por su parte, Los Arcos et al. (2017) examinaron la asociación de los esfuerzos respiratorios y musculares percibidos y la carga de entrenamiento asociada para monitorear los cambios en varios parámetros de aptitud aeróbica y rendimiento neuromuscular durante 32 semanas de entrenamiento de fútbol en jugadores profesionales jóvenes. Gil-Rey, Lezaun y Los Arcos (2015) sugieren que un volumen y un entrenamiento muscular y respiratorio pueden afectar la mejora de la aptitud aeróbica en jugadores de fútbol junior durante la temporada.

\subsection{INVESTIGACIONES RELACIONADAS CON MOTIVACIÓN, COHESIÓN GRUPAL, ESTRÉS Y ANSIEDAD}

Castro-Sánchez et al. (2019) exponen que la motivación y la ansiedad representan factores psicológicos fundamentales en el contexto deportivo, debido a su estrecha relación con el rendimiento. Los resultados de esta investigación (CASTRO-SÁNCHEZ et al., 2019) indican que los jugadores de fútbol tienen altos niveles de ansiedad, por ello, resulta importante prestar atención a las asociaciones existentes entre el clima motivacional percibido y los niveles de ansiedad de los futbolistas en las divisiones de fútbol inferiores, promoviendo climas motivacionales orientados a la tarea e interviniendo mediante el uso de técnicas de relajación, con el fin de para disminuir los niveles de ansiedad entre los futbolistas jóvenes. Otros autores (GONZÁLEZ-PONCE et al., 2018) mostraron que tanto la tarea como el conflicto de relación aumentaron significativamente con el tiempo. El análisis de modelado multinivel mostró que la estrategia del juego y las competencias de desarrollo de personajes predijeron negativamente los conflictos entre tareas y relaciones a nivel individual, mientras que la competencia de 
motivación también se agregó como un importante predictor de conflicto entre tareas a nivel de equipo. Por otra parte, la competencia técnica predijo positivamente el conflicto de tareas a nivel de equipo. Estos autores (GONZÁLEZ-PONCE et al., 2018) nos sugieren la importancia de la competencia de coaching en dinámicas de grupo en el deporte. Leo et al. (2019) hablan sobre la unión, confianza e intercambio de información en los clubes de primera y segunda división B. Los hallazgos resaltan la necesidad de desarrollar intervenciones integradas de dinámica de equipo, ya que la cohesión influye en la eficacia colectiva. Para Pulido et al. (2018) el ambiente de entrenamiento creado por el entrenador puede ser crucial para la satisfacción o la frustración de las necesidades psicológicas básicas y, como consecuencia, podría desempeñar un papel importante en el compromiso deportivo de los jóvenes futbolistas. Leo et al. (2016) mostraron que en los primeros seis meses de competición, la cohesión del equipo tenía mayor poder para predecir la eficacia colectiva al final de la liga. La eficacia colectiva estuvo más relacionada con el rendimiento del equipo que la cohesión grupal al final de la temporada. Esta investigación (LEO et al., 2016) concluyó que durante la pretemporada y al comienzo de la temporada, los entrenadores de deportes de equipo deberían centrarse en los aspectos sociales y de tareas, tanto individualmente como a nivel de grupo; esto mejoraría la percepción de la eficacia del equipo colectivo y conduciría a un mejor rendimiento del equipo.

Según Marholz et al. (2016) indican una relación entre el clima motivacional percibido y el compromiso con el juego. En cambio para Martínez-Alvarado, Guillén y Feltz (2016) la necesidad de autonomía es la variable que predice mejor los síntomas del agotamiento y el compromiso del atleta en los jugadores de fútbol de la tercera división española. Finalmente, el síntoma de satisfacción personal reducido fue la necesidad psicológica que mejor predijo los síntomas de agotamiento. Hevilla-Merino y Castillo-Rodríguez (2018) han contribuido a aumentar el conocimiento de la fluctuación de las características psicológicas que influyen en los jugadores de fútbol en los estados anteriores a la competencia y la influencia de la ansiedad cognitiva en la motivación. Los resultados obtenidos en esa investigación indicaron diferencias de motivación en ambas categorías y de concentración en la categoría sub16 entre estados basales y precompetitivos. Además, se encontró una relación inversa entre la motivación y la ansiedad cognitiva que puede ser un factor determinante de la atención en el fútbol.

Olmedilla et al. (2018) indicaron que los jugadores lesionados masculinos presentaron niveles de ansiedad más altos que aquellos que no estaban lesionados. En los datos de las jugadoras, los niveles de estrés de las no lesionadas son más altos que los de las jugadoras lesionadas. Con respecto a los deportistas no lesionados, los resultados mostraron que las jugadoras no lesionadas presentaron valores de ansiedad más altos que los de los jugadores masculinos no lesionados. Olmedilla, García-Alarcón y Ortega (2018) indicaron, a diferencia de investigaciones anteriores, que los jugadores no lesionados mostraron niveles más altos de estrés que los jugadores lesionados; además, dentro del grupo de jugadores lesionados, aquellos con lesiones moderadas mostraron niveles de estrés más altos que aquellos con lesiones leves, los que tenían lesiones graves mostraron niveles de estrés más altos que aquellos con lesiones moderadas y aquellos con lesiones muy graves reportaron niveles de estrés más altos que aquellos que tenían lesiones graves. Zurita-Ortega et al. (2017) analizaron la relación entre resiliencia, estados de ansiedad y lesiones deportivas, teniendo en cuenta el nivel de competencia. Los resultados de su estudio (ZURITA-ORTEGA et al., 2017) mostraron que el nivel competitivo afecta directamente la capacidad de enfrentar futuras lesiones independientemente de la ansiedad que tengan los jugadores. 
Usán et al. (2016) analizaron las relaciones entre motivación, compromiso deportivo y autoconcepto físico entre los futbolistas adolescentes de la provincia de Zaragoza. Los resultados mostraron diferencias significativas entre los distintos niveles de competitividad y dos perfiles de comportamiento: el primero caracterizado por un comportamiento más autodeterminado (motivación intrínseca, compromiso deportivo, disfrute deportivo, oportunidades de participación, competencia, confianza en sí mismo) y el segundo se distingue por un comportamiento menos adaptativo (motivación extrínseca, motivación, restricciones sociales o cuerpo atractivo entre otras). Pedreño et al. (2015) resaltaron la importancia de los objetivos sociales, los elogios por el comportamiento autónomo y los mediadores psicológicos para fomentar un mayor compromiso en los jóvenes futbolistas. Por su parte, Naveira (2018) expone que una alta autoeficacia favorece niveles más altos de rendimiento físico, y que la tendencia defensiva o de ataque del jugador es multifactorial (física, técnica, táctica y psicológica) y producto de su desarrollo histórico-deportivo (influencia del entrenador, filosofía del juego y demarcación). Rubio et al. (2018) sugieren que el discurso previo al juego contribuye a mejorar la autoeficacia de los deportistas. Los hallazgos de Fernández-Navarro et al. (2016) mostraron que el estilo de juego de un equipo se definía mediante indicadores de rendimiento específicos y, en consecuencia, los equipos se pueden organizar para crear un perfil de estilo de juego, además, los clubes podrían usar ejercicios de entrenamiento específicos dirigidos a mejorar sus estilos de juego.

\section{CONCLUSIONES}

Tras la revisión de los 54 artículos utilizados para esta revisión sobre la presencia de la mujer en los estudios sobre el fútbol español, los niños y fútbol y las variables psicosociales analizadas en los manuscritos publicados sobre fútbol español en los últimos 5 años (20152019) concluimos que,

1. El análisis de los distintos estudios relacionados con el fútbol en el contexto español, ayuda a identificar la diversidad de líneas de investigación relacionadas con la temática abordada y el grado de especificidad de las mismas, otorgando a la comunidad científica una visión global de la relevancia y cantidad de investigaciones realizadas hasta la fecha de aceptación de este trabajo.

2. Se halla un escaso número de publicaciones de fútbol en el ámbito femenino, así como estudios que reflejen la necesidad de abordar este deporte en el contexto educativo o de base como fin para promocionar unos hábitos saludables a edades tempranas.

3. En los estudios sobre niños y fútbol no se ha estudiado en profundidad el importante papel que desempeñan los padres, la importancia del juego limpio y la violencia en los estadios de fútbol, un tema que a menudo sale en los medios de comunicación españoles.

4. Los trabajos que han analizado las variables psicológicas y psicosociales en el fútbol español se centran en la motivación, la cohesión grupal, la autoeficacia y la ansiedad y autoconcepto.

5. Es necesario cubrir con mayor aportación científica el fútbol femenino y el fútbol en niños dada la gran cantidad de practicantes en España y su relevancia como visibilidad de la mujer en la participación deportiva y como un importante escenario para trabajar la educación de los niños y su componente social, afectivo y emocional, más allá de potenciar la esfera física del ser humano. 


\section{REFERENCIAS}

AGUILAR, Miguel. Temporal consistencies in two champion teams of European football?. Retos: nuevas tendencias en educación física, deporte y recreación, v. 34, p. 94-99, 2018.

ALBÓ, Javier. Metaphorical Language in the World of Soccer. Apunts. Educació Física i Esports, v. 129, p. 26-43, 2017.

AMATRIA, Mario; LAPRESA, Daniel; ARANA, Javier; ANGUERA, María Teresa; GARZÓN, Belén. Optimization of game formats in U-10 soccer using logistic regression analysis. Journal of Human Kinetics, v. 54, n. 1, p. 163-171, 2016. http://DOl:10.1515/hukin-2016-0047

AZCARATE, Unai; LOS ARCOS, Asier; YANCI, Javier. Effects of training programs integrated only with football drills on the cardiovascular and neuromuscular performance of amateur footballer players. Journal of Sport and Health Research, v. 10, n. 2, p. 257-268, 2018.

BALAGUER, Isabel; CASTILLO, Isabel; CUEVAS, Ricardo; ATIENZA, Francisco. The Importance of Coaches' Autonomy Support in the Leisure Experience and Well-Being of Young Footballers. Frontiers in Psychology, v. 9, 2018. http://doi:10.3389/fpsyg.2018.00840

BUNK, Brian. Sardinero and not a can of sardines: soccer and Spanish ethnic identities in New York City during the 1920s. Journal of Urban History, v. 41, n. 3, p. 444-459, 2015. https://doi. org/10.1177/0096144214566959

CABALLERO, Pablo; RUBIO, Javier; GODOY, Sergio José. Influence of situational variables on the U'18 soccer performance analysis. Retos: nuevas tendencias en educación física, deporte y recreación, v. 32, p. 224-227, 2017.

CASAMICHANA, David; BRADLEY, Paul; CASTELLANO, Julen. Influence of the Varied Pitch Shape on Soccer Players Physiological Responses and Time-Motion Characteristics During Small-Sided Games. Journal of Human Kinetics, v. 64, n. 1, p. 171-180, 2018. https://doi. org/10.1515/hukin-2017-0192

CASTELLANO, Julen; ECHEAZARRA, Ibon; ESTEFANO, Iban. Comparison of the physical demands in sub13 and sub14 football players on a 7-a-side game played with different lengths. Cultura Ciencia y Deporte, v. 12, n. 34, p. 55-65, 2017.

CASTRO-SÁNCHEZ, Manuel; ZURITA-ORTEGA, Félix; CHACÓN-CUBEROS, Ramón; LOZANO-SÁNCHEZ, Antonio. Motivational climate and levels of anxiety in soccer players of lower divisions. RETOS-Nuevas Tendencias en Educación Física, Deporte y Recreación, v. 35, p. 164-169, 2019.

CEJUDO, Antonio; ROBLES-PALAZÓN, Francisco Javier; AYALA, Francisco; CROIX, Mark; ORTEGA-TORO, Enrique; SANTONJA-MEDINA, Fernando; DE BARANDA, Pilar. Age-related differences in flexibility in soccer players 8-19 years old. PeerJ, v. 7, e6236, 2019.

CLEMENTE, José Antonio; MORENO-ARRONES, Luis Jesús; GIL, Salvador. Diferencias entre distintas orientaciones del espacio, relativizadas al perfil individual del jugador. RETOS-Nuevas Tendencias en Educación Física, Deporte y Recreación, v. 35, p. 3-6, 2019.

CONEJOS, Cristina; GINER, Antonio; VINUESA, Jordi; DEL CASTILLO, José Miguel. Improvement of soccer nutrition knowledge about carbohydrates with posters. Archivos de medicina del deporte: revista de la Federación Española de Medicina del Deporte y de la Confederación Iberoamericana de Medicina del Deporte, v. 165, p. 16-19, 2015. 
DEL COSO, Juan; HERRERO, Helena; SALINERO, Juan J.. Injuries in Spanish female soccer players. Journal of Sport and Health Science, v. 7, n. 2, p. 183-190, 2018. https://doi. org/10.1016/i.jshs.2016.09.002

DÍAZ-CIDONCHA, Jorge; REFOYO, Ignacio; CALLEJA-GONZÁLEZ, Julio; DELLAL, Alexandre. Comparison of tactical offensive variables in different playing surfaces in sided games in soccer. International Journal of Performance Analysis in Sport, v. 15, n. 1, p. 297-314, 2015. https://doi.org/10.1080/24748668.2015.11868794

DÍAZ-MIGUEL, Salvador; LOPEZOSA-RECA, Eva; BENHAMU-BENHAMU, Salomón; ORTEGAÁVILA, Ana Belén; GARCÍA-DE-LA-PEÑA, Raquel; GIJON-NOGUERON, Gabriel. Structural differences in the lower extremities in children aged 7-9 years, caused by playing football: $A$ cross-sectional study. The Foot, v. 34, p. 78-82, 2018. https://doi.org/10.1016/j.foot.2017.11.004

FERNÁNDEZ-NAVARRO, Javier; FRADUA, Luis; ZUBILLAGA, Asier; FORD, Paul R.; MCROBERT, Allistair. Attacking and defensive styles of play in soccer: analysis of Spanish and English elite teams. Journal of Sports Sciences, v. 34, n. 24, p. 2195-2204, 2016. https://doi.org 110.1080/02640414.2016.1169309

GARCÍA, Félix; SANTANA, Jorge. Exploring mental toughness in soccer players of different levels of performance. Revista iberoamericana de psicología del ejercicio y el deporte, v. 13, n. 2, p. 297-303, 2018.

GIL-REY, Erreka; LEZAUN, Alejandro; LOS ARCOS, Asier. Quantification of the perceived training load and its relationship with changes in physical fitness performance in junior soccer players. Journal of Sports Sciences, v. 33, n. 20, p. 2125-2132, 2015. https://doi.org/10.1080/02 $\underline{640414.2015 .1069385}$

GÓMEZ, Maite; ALFARO, Elida. El acceso de las mujeres al deporte profesional: el caso del fútbol. Femeris, v. 3, n 2, p. 178-180, 2018. https://doi.org/10.20318/femeris.2018.4325

GONZÁLEZ, Y; FERNÁNDEZ-ORTEGA, J.; GARAVITO-PEÑA, F. Características de fuerza y velocidad de ejecución en mujeres jóvenes futbolistas. Revista Internacional de Medicina de Ciencias de la Actividad Física y el Deporte, v. 19, n 73, p. 167-179, 2019. http://doi. org/10.15366/rimcafd2019.73.012

GONZÁLEZ-PONCE, Inmaculada; LEO, Francisco; JIMÉNEZ, Ruth; SÁNCHEZ-OLIVA, David; SARMENTO, Hugo; FIGUEIREDO, Antonio; GARCÍA-CALVO, Tomás. Athletes' perceptions of coaching competency and team conflict in sport teams: A multilevel analysis. European Journal of Sport Science, v. 18, n. 6, p. 851-860, 2018. https://doi.org/10.1080/17461391.2018.1461245

GUIRAO-GORIS, Josep Adolf; OLMEDO, Ángela; FERRER, Esperanza. El artículo de revisión. Revista Iberoamericana de Enfermeria Comunitaria, v.1, n.1, p.6, 2008. Disponible en: http:// revista.enfermeriacomunitaria.org/articuloCompleto.php?|D=7. Consultado el: 23 febr. 2019

GUTIÉRREZ, José V.; FERNÁNDEZ, Jorge del Rosario; PONCE, Jesús Gustavo; LAGARES, Carolina; DE CASTRO, Guillermo Ramón. Hamstring extensibility in female elite soccer players. Retos-nuevas tendencias en educación física deporte y recreación, v. 33, p. 175178, 2018.

HELLÍN, Marta; GARCÍA-JIMÉNEZ, José V.; GARCÍA-PELLICER, Juan J.. Intensity of Physical Education lessons in children according to the type of activity: soccer, badminton, aerobics and motor skills. Journal of Physical Education and Sport, v. 19, p. 148-155, 2019. http:// 
HEVILLA-MERINO, Álvaro; CASTILLO-RODRÍGUEZ, Alfonso. Fluctuation of the psychological characteristics of sports performance on young soccer players. Basal state vs. precompetitive. Cuadernos de Psicología del Deporte, v. 18, n. 3, p. 169-178, 2018.

IBÁÑEZ, Sergio José; PÉREZ-GOYE, José Antonio; COUREL-IBÁÑEZ, Javier; GARCÍARUBIO, Javier. The impact of scoring first on match outcome in women's professional football. International Journal of Performance Analysis in Sport, v. 18, n. 2, p. 318-326, 2018. https://doi.org/10.1080/24748668.2018.1475197

LANDERS, Joseph G.; ESCH, Tobias. Sport physiology, dopamine and nitric oxide-Some speculations and hypothesis generation. Medical Hypotheses, v. 85, n. 6, p. 905-909, 2015. https://doi.org/10.1016/j.mehy.2015.09.012

LAPRESA, Daniel; ARANA, Javier; AMATRIA, Mario; FERNÁNDEZ, Francisco javier; ANGUERA, María Teresa. Soccer: effects of a teaching unit in early initiation. Apunts. Educació Fisica i Esports, v. 127, p. 59-67, 2017.

LEO, Francisco M.; GONZÁLEZ-PONCE, Inmaculada; GARCÍA-CALVO, Tomás; SÁNCHEZOLIVA, David; FILHO, Edson. The relationship among cohesion, transactive memory systems, and collective efficacy in professional soccer teams: A multilevel structural equation analysis. Group Dynamics: Theory, Research, and Practice, v. 23, n. 1, p. 44, 2019. http:// dx.doi.org/10.1037/gdn0000097

LEO, Francisco M.; GONZALEZ-PONCE, Inmaculada; SANCHEZ-OLIVA, David; AMADO, Diana; GARCIA-CALVO, Tomás. Exploring direction between cohesion and collective efficacy and relationships with performance of football teams. South African Journal for Research in Sport, Physical Education and Recreation, v. 38, n. 3, p. 113-126, 2016.

LÓPEZ-FERNÁNDEZ, Jorge; GARCÍA-UNANUE, Jorge; SÁNCHEZ-SÁNCHEZ, Javier; LEÓN, Manuel; HERNANDO, Enrique; GALLARDO, Leonor. Neuromuscular responses and physiological patterns during a soccer simulation protocol. Artificial turf versus natural grass. The Journal of sports medicine and physical fitness, v. 58, n. 11, p. 1602-1610, 2018. http://10.23736/S0022-4707.17.07768-4

LÓPEZ-MARTÍNEZ, Raúl; FERNÁNDEZ-FERNÁNDEZ, José Luis. Corporate Social Responsibility and Good Governance in spanish football clubs. UCJC Business and Society Review, v. 46, p. 38-53, 2015.

LÓPEZ-VALENCIANO, Alejandro; AYALA, Francisco; VERA-GARCÍA, Francisco J.; DE STE, Mark; HERNÁNDEZ-SÁNCHEZ, Sergio; RUIZ-PÉREZ, Iñaki; SANTONJA, Fernando. Comprehensive profile of hip, knee and ankle ranges of motion in professional football players. The Journal of Sports Medicine and Physical Fitness, v. 59, n. 1, p. 102-109, 2019.

LOS ARCOS, Asier; MARTÍNEZ-SANTOS, Raúl; YANCI, Javier; MÉNDEZ-VILLANUEVA, Alberto. Monitoring perceived respiratory and muscular exertions and physical fitness in young professional soccer players during a 32-week period. Kinesiology: International Journal of Fundamental and Applied Kinesiology, v. 49, n. 2, p. 153-160, 2017. https://doi. org/10.26582/k.49.2.12

LOS ARCOS, Asier; VÁZQUEZ, Juan Sebastián; MARTÍN, Juan; LERGA, Javier; SÁNCHEZ, Felipe; VILLAGRA, Federico; ZULUETA, Javier J. Effects of small-sided games vs. interval training in aerobic fitness and physical enjoyment in young elite soccer players. PloS one, v. 10, n. 9, 2015. https://doi.org/10.1371/journal.pone.0137224 
LOZANO-BERGES, Gabriel; MATUTE-LLORENTE, Ángel; GÓMEZ-BRUTON, Alejandro; GONZÁLEZ-AGÜERO, Alex; VICENTE-RODRIGUEZ, Germán; CASAJÚS, José A.. Bone geometry in young male and female football players: a peripheral quantitative computed tomography (pQCT) study. Archives of Osteoporosis, v. 13, n. 1, p. 57, 2018. https://doi. org/10.1007/s11657-018-0472-2

LLOPIS-GOIG, Ramón; FLORES, Helena. The creation of female supporter communities: A challenge to the configuration of the sex/gender system which characterise Spanish football? AIBR Revista de Antropologia Iberoamericana, v. 12, n 3, p. 411-433, 2017. https://doi. org/10.1057/978-1-137-59025-1 12

MANEIRO, Rubén; AMATRIA, Mario. Polar Coordinate Analysis of Relationships With Teammates, Areas of the Pitch, and Dynamic Play in Soccer: A Study of Xabi Alonso. Frontiers in Psychology, v. 9, p. 389, 2018. https://doi.org/10.3389/fpsyg.2018.00389

MANEIRO, Rubén; AMATRIA, Mario; ANGUERA, María Teresa. Dynamics of Xavi Hernández's game: A vectorial study through polar coordinate analysis. Proceedings of the Institution of Mechanical Engineers, Part P: Journal of Sports Engineering and Technology. 2019. https:// doi.org/10.1177/1754337119830472

MARHOLZ, Paula; GÓMEZ-LÓPEZ, Manuel; MARTÍN, Ignacio; GARRIDO, Rafael Enrique; GARCIA-MAS, Alexandre; CHIROSA, L. Javier. Role played by the coach in the adolescent players'commitment. Studia Psychologica, v. 58, n. 3, p. 184, 2016. http://DOl:10.21909/ sp.2016.03.716

MARTíNEZ-ALVARADO, Julio Román; GUILLÉN, Félix; FELTZ, Deborah. (2016). Athletes' motivational needs regarding burnout and engagement. Revista de Psicologia del Deporte, v. 25, n. 1, p. 65-71, 2016.

MOSCOSO-SÁNCHEZ, David; RODRÍGUEZ-DÍAZ, Álvaro; FERNÁNDEZ-GAVIRA, Jesús. Elitist rhetoric and the sports gap. Examining the discourse and reality of sport in Spain. European Journal for Sport and Society, v. 12, n. 1, p. 31-51, 2015. https://doi.org/10.1080/16138171.2015 .11687955

NAVEIRA, Alejo. Autoeficacia y rendimiento en jugadores de fútbol. Cuadernos de Psicología del Deporte, v. 18, n. 2, p. 66-78, 2018.

NEVADO-GARROSA, Fabio; TEJERO-GONZÁLEZ, Carlos María; PAREDES-HERNÁNDEZ, Víctor; CAMPO-VECINO, Juan. Análisis comparativo de las demandas físicas de dos tareas de juego reducido en fútbol profesional. Archivos de Medicina del Deporte, v. 32, n. 2, p. 82-86, 2015.

OLMEDILLA, Aurelio; GARCÍA-ALARCÓN, M.; ORTEGA, Enrique. Relationships between sports injuries and stress in female football and indoor football. Journal of Sport and Health Research, v. 10, n. 3, p. 339-348, 2018.

OLMEDILLA, Aurelio; ORTEGA, Enrique; ROBLES-PALAZÓN, Francisco Javier; SALOM, Miquel; GARCÍA-MAS, Alexandre. Healthy Practice of Female Soccer and Futsal: Identifying Sources of Stress, Anxiety and Depression. Sustainability, v. 10, n. 7, p. 2268, 2018. https://doi. org/10.3390/su10072268

ORTEGA-TORO, Enrique; GARCÍA-ANGULO, Antonio; GIMÉNEZ-EGIDO, José María; GARCÍA-ANGULO, Francisco J.; PALAO, José. Effect of modifications in rules in competition on participation of male youth goalkeepers in soccer. International Journal of Sports Science \&

Coaching, v. 13, n. 6, p. 1040-1047, 2018. https://doi.org/10.1177/1747954118769423 
PASCUAL, Norberto; CARBONELL, José Antonio. Analysis of technical and tactical principles in women's soccer. Journal of Physical Education and Sport, v. 18, n. 3, p. 1488 - 1494, 2018. http://DOI:10.7752/jpes.2018.03219

PEDREÑO, Noelia; FERRIZ-MOREL, Roberto; RIVAS, Shai; ALMAGRO, Bartolomé; SÁENZLÓPEZ, Pedro; CERVELLÓ, Eduardo; MORENO-MURCIA, Juan Antonio.. Sport commitment in adolescent soccer players. Motricidade, v. 11, n. 4, p. 3-14, 2015. https://doi.org/10.6063/ motricidade.2969

PEÑA-GONZÁLEZ, Iván; FERNÁNDEZ-FERNÁNDEZ, Jaime; MOYA-RAMÓN, Manuel; CERVELLÓ, Eduardo. Relative age effect, biological maturation, and coaches' efficacy expectations in young male soccer players. Research quarterly for exercise and sport, v. 89, n. 3, p. 373-379, 2018. https://doi.org/10.1080/02701367.2018.1486003

PÉREZ, Salvador; CASTAÑO, Raimundo; SÁNCHEZ, Antonio; RODRÍGUEZ, Alberto; MENA, José Manuel; FUENTES, José María; CASTAÑO, Celia. Why do I play football if I am a woman?: Motivations to play football. RETOS-Nuevas Tendencias en Educación Física, Deporte y Recreación, v. 34, p. 183-188, 2018.

PRADO-ROMÁN, Miguel; PRADO-ROMÁN, Alberto; PLAZA-CASADO, Paola; PAZ-GIL, Iria. Public's Behaviour in Front of Sports: Case of Spanish Football. In: ANNUAL MEETING OF THE EUROPEAN ACADEMY OF MANAGEMENT AND BUSINESS ECONOMICS. Cham: Springer, Sept. 2017. p. 214-224.

PRÁXEDES, Alba; MORENO, Alberto; GARCÍA-GONZÁLEZ, Luis; PIZARRO, David; DEL VILLAR, Fernando. The Relative Age Effect on soccer players in formative stages with different sport expertise levels. Journal of Human Kinetics, v. 60, n. 1, p. 167-173, 2017. http:// DOl:10.1515/hukin-2017-0100

PULIDO, Juan J.; SÁNCHEZ-OLIVA, David; SÁNCHEZ-MIGUEL, Pedro A.; AMADO, Diana; GARCÍA-CALVO, Tomás. Sport commitment in young soccer players: A self-determination perspective. International Journal of Sports Science \& Coaching, v. 13, n. 2, p. 243-252, 2018. https://doi.org/10.1177/1747954118755443

RUBIO, Víctor J.; HERNÁNDEZ, José; SÁNCHEZ-IGLESIAS, Iván; CANO, Alicia; BUREO, Rafael. The effects of coaches' Pre-game Speeches on young players' self-efficay. Revista de Psicología del Deporte, v. 27, n. 1, p. 59, 2018.

SARMENTO, Hugo; ANGUERA, María Teresa; PEREIRA, Antonio; ARAÚJO, Duarte. Talent identification and development in male football: A systematic review. Sports Medicine, v. 48, n. 4, p. 907-931, 2018. https://doi.org/10.1007/s40279-017-0851-7

SEDANO, Silvia; VAEYENS, Roel; REDONDO, Juan Carlos. The relative age effect in Spanish female soccer players. Influence of the competitive level and a playing position. Journal of Human Kinetics, v. 46, n. 1, p. 129-137, 2015. http://DOl:10.1515/hukin-2015-0041

SERRA-OLIVARES, Jaime; GARCÍA-LÓPEZ, Luis Miguel; CALDERÓN, Antonio. Learning and talent in soccer: Aprendizaje y talento en fútbol. Apunts. Educación Física y Deportes, v. 129, p. 64-77, 2017.

SEVIL, Javier; PRÁXEDES, Alba; GARCÍA-GONZÁLEZ, Luis; MORENO, Alberto; DEL VILLAR, Fernando. Evolution of tactical behavior of soccer players across their development. International Journal of Performance Analysis in Sport, v. 17, n. 6, p. 885-901, 2017. https://doi.org/10.1080/24748668.2017.1406781 
SIMÓN, Juan Antonio. Football, newspapers and national identities in Spain, 1975-

2017. Movimento, v. 24, n. 4, p. 1235-1248, 2018. https://doi.org/10.22456/1982-8918.81403

SOUZA, Djalma Oliveira de; ESPIRITO SANTO, Maria do; RIBEIRO, Rosa Cavalcante. Mulheres no mundo do futebol: representações. Revista Brasileira de Futsal e Futebol, v. 10, n. 41, p. 763773, 2018.

TÉLLEZ-GIRÓN, Jonathan; ALVARADO, Matías. Concurrency simulation in soccer. In: INTERNATIONAL CONFERENCE ON SOCIAL ROBOTICS. Cham: Springer, Nov. 2016. p. $961-$ 970.

USÁN, Pablo; SALAVERA, Carlos; MURILLO, Víctor; MEGÍAS, Juan José. Relationship between motivation, commitment and selfconcept in adolescent soccer players of different sport categories. Cuadernos de Psicología del Deporte, v. 16, n. 1, p. 199-209, 2016.

VACZI, Mariann. Soccer, culture and society in Spain: an ethnography of Basque fandom. New Yook: Abingdon Routledge, 2015.

ZAMBOM-FERRARESI, Fabiola; RIOS, Vicente; LERA-LÓPEZ, Fernando. Determinants of sport performance in European football: What can we learn from the data?. Decision Support Systems, v. 114, p. 18-28, 2018. https://doi.org/10.1016/..dss.2018.08.006

ZAMBOM-FERRARESI, Fabiola; GARCÍA-CEBRIÁN, Lucía; LERA-LÓPEZ, Fernando. Competitive balance in male and female leagues: Approximation to the Spanish case. Journal of Physical Education and Sport v. 18, p. 1323-1329, 2018. https://DOl:10.7752/jpes.2018.s3196

ZURITA-ORTEGA, Félix; RODRÍGUEZ-FERNÁNDEZ, Sonia; OLMO-EXTREMERA, Marta; CASTRO-SÁNCHEZ, Manuel; CHACÓN-CUBEROS, Ramón; CEPERO-GONZÁLEZ, María del Mar. Analysis of resilience, anxiety and sports injuries in soccer by competition level. Cultura Ciencia y Deporte, v. 12, n. 35, p. 135-142, 2017. 